\title{
A Supplement to Self-Organization Theory of Dreaming
}

\author{
Wei Zhang * \\ School of psychology, Nanjing Normal University, Nanjing, China
}

Keywords: self-organization, dreaming, memory consolidation, two-stage model, by-product

\section{DREAMING: A PROCESS OF SELF-ORGANIZATION}

Kahn and Hobson (1993) proposed that dreams are a product of self-organization of brain during sleep. As a complex system far from equilibrium state, the dreaming brain may form a new pattern by the interaction between components within this system. At REM sleep stage, signals from neuronal clusters self-organize and form image fragments, then the image fragments interact and produce images, and finally these materials are associated into a relatively continuous narrative (i.e., dreams).

This process above happens under a weak control of brain, and the conditions of this state mainly include: (a) the inhibition of external stimuli or feedback; (b) the changes of neuromodulatory systems (e.g., the governance of aminergic neurochemicals and the weakness of cholinergic neurochemicals); (c) the bombardment of PGO wave to the visual cortex; and (d) the changes of neural activity in brain (e.g., the activation of limbic system and the reduction of prefrontal regions). In this situation, the brain could focus on the internal world and integrate various psychological and physiological changes into dream content (Kahn et al., 2000, 2002).

However, self-organization mechanism could not determine which memories will be activated and incorporated into dream content (Kahn, 2013), although it provides an approach how an ordered structure, behavior or pattern spontaneously emerges from the interaction between components or subsystems without an external guidance (see Isaeva, 2012; Sasai, 2013). It merely combines materials which present during sleep and then makes up a "story." Thus, the content of dreams is, to a large degree, determined by the active materials in sleep. In this article, we attempt to make a supplement to this theory on the basis of the two-stage model of memory consolidation.

\section{DREAMING AND MEMORY CONSOLIDATION}

Memory is a major element of dreams. The Continuity Hypothesis $(\mathrm{CH})$ considers that the content of dreams is a reflection of waking life, and the former is also carried over into the latter (Domhoff, 1996). Thus, the episodic memories of real life will replay during sleep. This viewpoint has been supported by many investigations about dreams that contain waking experiences (e.g., Domhoff, 2001, 2003; Schredl and Piel, 2005; Pesant and Zadra, 2006).

However, there are several problems that the Continuity Hypothesis could not explain. Firstly,

Received: 30 November 2015 Accepted: 22 February 2016 Published: 08 March 2016

Citation:

Zhang W (2016) A Supplement to Self-Organization Theory of Dreaming.

Front. Psychol. 7:332. doi: 10.3389/fpsyg.2016.00332 according to this theory, major daily activities, such as highly focused cognitive processes (e.g., writing, reading, and arithmetic), will present in dream content, but the consequence is not (e.g., Hartmann, 2000). Secondly, although experiences of waking life could be found in dreams, the incorporated components in dreams are usually fragments rather than a whole (Fosse et al., 2003). This means real life is not entirely reply in dreams (e.g., Schwartz, 2003; Hartmann, 2010). Hence, the Continuity Hypothesis seems not to be the ultimate answer. 
An alternative explanation may be found in studies about the relationship between sleep and memory consolidation. According to the two-stage memory model, memory consolidation during sleep mainly contains a series of process as follows (McClelland et al., 1995; Nielsen and Stenstrom, 2005; Diekelmann and Born, 2010; Lewis and Durrant, 2011; Born and Wilhelm, 2012). (a) Newly acquired memories will be reactivated in NREM sleep, especially in SWS (Born and Wilhelm, 2012; Wamsley, 2014). In this period, there will be a mechanism that selects and determines which memories should be strengthened or weakened. If these memories are useful for the individual, they will be enhanced. If not (i.e., these memories are non-adaptive), they will be eliminated or fade away (Stickgold and Walker, 2013). Thus, this stage which mainly occurs in hippocampus will refer to a procedure of abstraction and extraction, and it leads to fragments of memory. (b) The temporary stored memories in hippocampus will be transferred into long-term memory which stores in neocortex about 6-7 days later. This stage needs to integrate these temporary memories into existing schema. Thus, it is associated with a course of redistributing memories and usually results in new connections (Hartmann, 2010).

During these processes, the role of NREM sleep and that of REM sleep are not the same; they seem to be complementary (Diekelmann and Born, 2010; Rasch and Born, 2013). Furthermore, these two stages of sleep are involved with different types of memory. For instance, NREM sleep is more closely related to declarative memory, while REM sleep is mostly correlated with emotional memory and memory that participates in implicit learning (Rauchs et al., 2005; Smith, 2010). This could be a reason why dream content in NREM sleep is different from that in REM sleep.

Therefore, memories will be fragmentized in order to extract important information (e.g., newly encoded and emotional memories) during sleep, and these important information are preferentially activated and incorporated into dream content. They mainly contain: (a) new learning experiences (Born and Wilhelm, 2012; Wamsley, 2014) which need to be consolidated and will be involved with a process of reactivation. Evidences could be found in many studies about dream reports (e.g., Stickgold et al., 2000; Wamsley et al., 2010a,b) and preferential reactivation of corresponding brain regions (e.g., Peigneux et al., 2004; Rasch et al., 2007; Rudoy et al., 2009; O’Neill et al., 2010; Bendor and Wilson, 2012) after learning tasks; (b) memories that will be integrated into long-term memory. This procedure is mainly reflected in dream-lag effect in REM sleep (Nielsen et al., 2004; Nielsen and Stenstrom, 2005; Blagrove et al., 2011; van Rijn et al., 2015); (c) long-term memories that are compatible with newly stored memories. As the former has to make connections with the latter in order to form a new cognitive schema (Lewis and Durrant, 2011), this process will be involved with remote memories.

As a consequence, by organizing these different fragments of memory, dreams could give rise to new connections and form a novel "story" that we have not experienced before, even exhibit a scene that never happens in real life. However, memories which will be weaken or faded away may be also a component of dreams, so experiences that are selectively incorporated into dream content need more investigations.

\section{THE STRENGTHS OF SELF-ORGANIZATION THEORY OF DREAMING}

Taking account of the process of memory consolidation based on the two-stage model, self-organization theory of dreaming can explain many findings about dreams. Now we summarize them as follows.

(1) It explains the relationship between dreaming and memory consolidation. The procedure of memory consolidation during sleep is involved with reactivation, abstraction, and extraction, which results in fragments of memory. Then these fragments could be a source of dreams. When selforganization process occurs, these materials are gradually combined into a narrative. Hence, dreams seem to be a by-product of brain neural activity and reflect memory consolidation during sleep. This point is similar with the model of Murkar et al. (2014).

(2) It offers an explanation why dreams are easy to forget. As a result of dreaming brain's self-organization under a weak control, the combination of fragments (i.e., dreams) is just a temporary pattern. Thus, when the dreamer wakes and the control of brain revives, this impermanent stable state will disappear in a short time, and then the forgetting of dreams happens.

(3) Incongruity and discontinuity are universal in dream content, as the nature of dreams is a combination of fragments. Although the narrative of dreams is relatively continuous due to self-organization, it would not be a "perfect" story. Thus, these defective connections between fragments will be inevitably presented in dream content. In addition, other factors during sleep will also disturb its continuity, such as PGO wave (Kahn and Hobson, 1993; Kahn et al., 2000, 2002).

(4) This theory seems more reasonable than the Continuity Hypothesis. The Continuity Hypothesis considers that the content of dreams reflects a replay of waking life experiences, but investigations reveal that not everything happens in real life will be presented in dreams. In addition, the parts of incorporation are usually fragments rather than a complete scene. Hence, waking life experiences are selectively incorporated in dream content, and this procedure could be interpreted by memory consolidation.

(5) It could explain some of the distinctions between dreams of REM sleep and those of NREM sleep (see Hobson et al., 2000). Firstly, the frequency of dreaming in REM sleep is higher than that in NREM sleep, because the brain's status of the former is more suitable for dreaming, such as neurochemical modulation, the activity level of prefrontal regions and limbic system, and PGO wave (Kahn et al., 2002). Secondly, the dream content of REM sleep is more novel and less associated with waking life compared with that of NREM sleep, as the processing of memories during 
REM sleep is more related to remote memories and usually makes new connections, while dreams of NREM sleep depend on the reactivation of newly encoded memories in waking life. Furthermore, the processing of different types of memory could also be a factor that leads to a difference of dream content between these two sleep stages.

(6) From this perspective, the "simulation function" of dreams is merely a possibility that combines different information presented during sleep, and this "creative product" may provide an enlightenment to the dreamer. Several theories suggest that dreams may have simulation function, such as threat simulation (Revonsuo, 2000; Valli and Revonsuo, 2009), social interaction simulation (Brereton, 2000; McNamara et al., 2005) and protoconsciousness simulation (Hobson, 2009). Therefore, these researchers consider that dreams are an adaptive strategy for the individual, as it could increase the possibility of survival or develop mental function. However, the self-organization theory implies that dreams may be not functional themselves.

(7) Self-organization theory of dreaming could be compatible with various kinds of psychological and physiological changes during sleep. Except for memories and PGO wave, some other factors, such as emotions (e.g., Yu, 2007; Vandekerckhove and Cluydts, 2010; Malinowski and Horton, 2014) and external stimuli (e.g., Nielsen, 1993; Schredl et al., 2009; Bloxham and Durrant, 2014), could impact on the content of dreams. Therefore, it could

\section{REFERENCES}

Bendor, D., and Wilson, M. A. (2012). Biasing the content of hippocampal replay during sleep. Nat. Neurosci. 15, 1439-1444. doi: 10.1038/nn.3203

Blagrove, M., Henley-Einion, J., Barnett, A., Edwards, D., and Heidi Seage, C. (2011). A replication of the 5-7day dream-lag effect with comparison of dreams to future events as control for baseline matching. Conscious. Cogn. 20, 384-391. doi: 10.1016/j.concog.2010.07.006

Bloxham, A., and Durrant, S. (2014). The effect of external stimuli on dreams, as assessed using Q-Methodology. Intern. J. Dream Res. 7, 129-140. doi: 10.11588/ijodr.2014.2.15754

Born, J., and Wilhelm, I. (2012). System consolidation of memory during sleep. Psychol. Res. 76, 192-203. doi: 10.1007/s00426-011-0335-6

Brereton, D. P. (2000). Dreaming, adaptation, and consciousness. The social mapping hypothesis. Ethos 28, 379-409. doi: 10.1525/eth.2000. 28.3.379

Diekelmann, S., and Born, J. (2010). The memory function of sleep. Nat. Rev. Neurosci. 11, 114-126. doi: 10.1038/nrn2762

Domhoff, G. W. (1996). Finding Meaning in Dreams: A Quantitative Approach. New York, NY: Plenum Press.

Domhoff, G. W. (2001). A new neurocognitive theory of dreams. Dreaming 11, 13-33. doi: 10.1023/A:1009464416649

Domhoff, G. W. (2003). The Scientific Study of Dreams: Neural Networks, Cognitive Development, and Content Analysis. Washington, DC: American Psychological Association.

Fosse, M. J., Fosse, R., Hobson, J. A., and Stickgold, R. J. (2003). Dreaming and episodic memory: a functional dissociation? J. Cogn. Neurosci. 15, 1-9. doi: $10.1162 / 089892903321107774$

Hartmann, E. (2000). We do not dream of the 3 R's: implications for the nature of dreaming mentation. Dreaming 10, 103-110. doi: 1023/A:10094008 05830 also interpret special types of dreams (e.g., bad dreams, recurrent dreams, and nightmares). As all of these elements could be incorporated into dreams through self-organization mechanism, this theory provides a compatible framework that covers different sources of dreams.

\section{CONCLUSION}

Self-organization mechanism could offer a compatible framework that integrates different elements (e.g., memories, emotions, and external stimuli) into dreams. The two-stage model of memory consolidation could provide a gist how memories are abstracted, exacted and stored during sleep, and thereby become a source of dreams. From this perspective, dreams are a by-product of corresponding neural activity in sleeping brain. They reflect the function of NREM sleep and REM sleep, but they seem not to be functional themselves (Wamsley, 2014).

However, although self-organization theory of dreaming is compatible with most of findings about dreams, it is not easy to test. Furthermore, the incorporation rate of waking experiences is still obscure. Thus, this viewpoint needs more empirical evidences to support and enrich.

\section{AUTHOR CONTRIBUTIONS}

The author confirms being the sole contributor of this work and approved it for publication.
Hartmann, E. (2010). The dream always makes new connections: the dream is a creation, not a replay. Sleep Med. Clin. 5, 241-248. doi: 10.1016/j.jsmc.2010.01.009

Hobson, J. A. (2009). REM sleep and dreaming: towards a theory of protoconsciousness. Nat. Rev. Neurosci. 10, 803-813. doi: 10.1038/nrn2716

Hobson, J. A., Pace-Schott, E. F., and Stickgold, R. (2000). Dreaming and the brain: toward a cognitive neuroscience of conscious states. Behav. Brain Sci. 23, 793-842. doi: 10.1017/S0140525X00003976

Isaeva, V. (2012). Self-organization in biological systems. Biol. Bull. 39, 110-118. doi: $10.1134 /$ S1062359012020069

Kahn, D. (2013). Brain basis of self: self-organization and lessons from dreaming. Front. Psychol. 4:408 doi: 10.3389/fpsyg.2013.00408

Kahn, D., Combs, A., and Krippner, S. (2002). Dreaming as a function of chaos-like stochastic processes in the self-organizing brain. Nonlin. Dynam. Psychol. Life Sci. 6, 311-322. doi: 10.1023/A:1019758527338

Kahn, D., Krippner, S., and Combs, A. (2000). Dreaming and the self-organizing brain. J. Consciousn. Stud. 7, 4-11.

Kahn, D., and Hobson, J. A. (1993). Self-organization theory of dreaming. Dreaming 3, 151-178. doi: 10.1037/h0094378

Lewis, P. A., and Durrant, S. J. (2011). Overlapping memory replay during sleep builds cognitive schemata. Trends Cogn. Sci. 15, 343-351. doi: 10.1016/j.tics.2011.06.004

Malinowski, J., and Horton, C. L. (2014). Evidence for the preferential incorporation of emotional waking-life experiences into dreams. Dreaming 24, 18-31. doi: 10.1037/a0036017

McClelland, J. L., McNaughton, B. L., and O'Reilly, R. C. (1995). Why there are complementary learning systems in the hippocampus and neocortex: insights from the successes and failures of connectionist models of learning and memory. Psychol. Rev. 102, 419-457. doi: 10.1037/0033-295X.102.3.419

McNamara, P., McLaren, D., Smith, D., Brown, A., and Stickgold, R. (2005). A "Jekyll and Hyde" within aggressive versus friendly interactions in 
REM and non-REM dreams. Psychol. Sci. 16, 130-136. doi: 10.1111/j.09567976.2005.00793.x

Murkar, A., Smith, C., Dale, A., and Miller, N. (2014). A neuro-cognitive model of sleep mentation and memory consolidation. Intern. J. Dream Res. 7, 85-89. doi: 10.11588/ijodr.2014.1.10306

Nielsen, T. A. (1993). Changes in the kinesthetic content of dreams following somatosensory stimulation of leg muscles during REM sleep. Dreaming 3, 99-113. doi: 10.1037/h0094374

Nielsen, T. A., Kuiken, D., Alain, G., Stenstrom, P., and Powell, R. A. (2004). Immediate and delayed incorporations of events into dreams: further replication and implications for dream function. J. Sleep Res. 13, 327-336. doi: 10.1111/j.1365-2869.2004.00421.x

Nielsen, T. A., and Stenstrom, P. (2005). What are the memory sources of dreaming? Nature 437, 1286-1289. doi: 10.1038/nature04288

O'Neill, J., Pleydell-Bouverie, B., Dupret, D., and Csicsvari, J. (2010). Play it again: reactivation of waking experience and memory. Trends Neurosci. 33, 220-229. doi: 10.1016/j.tins.2010.01.006

Peigneux, P., Laureys, S., Fuchs, S., Collette, F., Perrin, F., Reggers, J., et al. (2004). Are spatial memories strengthened in the human hippocampus during slow wave sleep? Neuron 44, 535-545. doi: 10.1016/j.neuron.2004.10.007

Pesant, N., and Zadra, A. (2006). Dream content and psychological well-being: a longitudinal study of the continuity hypothesis. J. Clin. Psychol. 62, 111-121. doi: $10.1002 /$ jclp.20212

Rasch, B., Büchel, C., Gais, S., and Born, J. (2007). Odor cues during slow-wave sleep prompt declarative memory consolidation. Science 315, 1426-1429. doi: 10.1126/science. 1138581

Rasch, B., and Born, J. (2013). About sleep's role in memory. Physiol. Rev. 93, 681-766. doi: 10.1152/physrev.00032.2012

Rauchs, G., Desgranges, B., Foret, J., and Eustache, F. (2005). The relationships between memory systems and sleep stages. J. Sleep Res. 14, 123-140. doi: 10.1111/j.1365-2869.2005.00450.x

Revonsuo, A. (2000). The reinterpretation of dreams: an evolutionary hypothesis of the function of dreaming. Behav. Brain Sci. 23, 793-1121. doi: 10.1017/S0140525X00004015

Rudoy, J. D., Voss, J. L., Westerberg, C. E., and Paller, K. A. (2009). Strengthening individual memories by reactivating them during sleep. Science 326, 1079. doi: $10.1126 /$ science. 1179013

Sasai, Y. (2013). Cytosystems dynamics in self-organization of tissue architecture. Nature 493, 318-326. doi: 10.1038/nature11859

Schredl, M., Atanasova, D., Hörmann, K., Maurer, J. T., Hummel, T., and Stuck, B. A. (2009). Information processing during sleep: the effect of olfactory stimuli on dream content and dream emotions. J. Sleep Res. 18, 285-290. doi: 10.1111/j.1365-2869.2009.00737.x
Schredl, M., and Piel, E. (2005). Gender differences in dreaming: are they stable over time? Pers. Individ. Dif. 39, 309-316. doi: 10.1016/j.paid.2005.01.016

Schwartz, S. (2003). Are life episodes replayed during dreaming? Trends Cogn. Sci. 7, 325-327. doi: 10.1016/S1364-6613(03)00162-1

Smith, C. (2010). Sleep states, memory processing, and dreams. Sleep Med. Clin. 5, 217-228. doi: 10.1016/j.jsmc.2010.01.002

Stickgold, R., Malia, A., Maguire, D., Roddenberry, D., and O'Connor, M. (2000). Replaying the game: hypnagogic images in normals and amnesics. Science 290, 350-353. doi: 10.1126/science.290.5490.350

Stickgold, R., and Walker, M. P. (2013). Sleep-dependent memory triage: evolving generalization through selective processing. Nat. Neurosci. 16, 139-145. doi: $10.1038 / \mathrm{nn} .3303$

Valli, K., and Revonsuo, A. (2009). The threat simulation theory in light of recent empirical evidence: a review. Am. J. Psychol. 122, 17-38.

van Rijn, E., Eichenlaub, J. B., Lewis, P. A., Walker, M. P., Gaskell, M. G., Malinowski, J. E., et al. (2015). The dream-lag effect: selective processing of personally significant events during Rapid Eye Movement sleep, but not during Slow Wave Sleep. Neurobiol. Learn. Mem. 122, 98-109. doi: 10.1016/j.nlm.2015.01.009

Vandekerckhove, M., and Cluydts, R. (2010). The emotional brain and sleep: an intimate relationship. Sleep Med. Rev. 14, 219-226. doi: 10.1016/j.smrv.2010.01.002

Wamsley, E. J. (2014). Dreaming and offline memory consolidation. Curr. Neurol. Neurosci. Rep. 14, 1-7. doi: 10.1007/s11910-013-0433-5

Wamsley, E. J., Perry, K., Djonlagic, I., Reaven, L. B., and Stickgold, R. (2010a). Cognitive replay of visuomotor learning at sleep onset: temporal dynamics and relationship to task performance. Sleep 33, 59-68.

Wamsley, E. J., Tucker, M., Payne, J. D., Benavides, J. A., and Stickgold, R. (2010b). Dreaming of a learning task is associated with enhanced sleep-dependent memory consolidation. Curr. Biol. 20, 850-855. doi: 10.1016/j.cub.2010.03.027

Yu, C. K. (2007). Emotions before, during, and after dreaming sleep. Dreaming 17, 73-86. doi: 10.1037/1053-0797.17.2.73

Conflict of Interest Statement: The author declares that the research was conducted in the absence of any commercial or financial relationships that could be construed as a potential conflict of interest.

Copyright (C) 2016 Zhang. This is an open-access article distributed under the terms of the Creative Commons Attribution License (CC BY). The use, distribution or reproduction in other forums is permitted, provided the original author(s) or licensor are credited and that the original publication in this journal is cited, in accordance with accepted academic practice. No use, distribution or reproduction is permitted which does not comply with these terms. 